\title{
MRI Bone Segmentation Using Deformable Models and Shape Priors
}

\author{
Jérôme Schmid and Nadia Magnenat-Thalmann \\ MIRALab, University of Geneva, CH-1211 Geneva, Switzerland \\ \{schmid, thalmann\}@miralab.unige.ch
}

\begin{abstract}
This paper addresses the problem of automatically segmenting bone structures in low resolution clinical MRI datasets. The novel aspect of the proposed method is the combination of physically-based deformable models with shape priors. Models evolve under the influence of forces that exploit image information and prior knowledge on shape variations. The prior defines a Principal Component Analysis (PCA) of global shape variations and a Markov Random Field (MRF) of local deformations, imposing spatial restrictions in shapes evolution. For a better efficiency, various levels of details are considered and the differential equations system is solved by a fast implicit integration scheme. The result is an automatic multilevel segmentation procedure effective with low resolution images. Experiments on femur and hip bones segmentation from clinical MRI depict a promising approach (mean accuracy: $1.44 \pm 1.1 \mathrm{~mm}$, computation time: $2 \mathrm{mn} 43 \mathrm{~s})$.
\end{abstract}

\section{Introduction}

Musculoskeletal disabilities seriously affect the majority of individuals over the age of 50. Osteoarthritis (OA) is often at origin of these disabilities, and its typical symptoms are inflammation, stiffness, pain and loss of mobility [1. Morphological analysis [2] of (changes in) organ shapes is precious in understanding which factors (e.g., impingements) can lead to serious OA. Automatic bones segmentation can be hence used to substitute or expedite tedious manual delimitations from medical images. Moreover, bone segmentation can serve as a basis for more advanced modeling of other essential structures, such as cartilages [3] or muscles 4 .

MRI is a flexible and non invasive modality. But bone segmentation can be challenging from clinical MRI images that suffer from poor image quality (imposed by time and clinical restrictions). Furthermore, bone intensity is not homogeneous in MRI due to differences in cortical and trabecular bones. This can affect some segmentation approaches (e.g., 56]). The use of prior knowledge considerably improves the robustness and the quality of segmentation especially when image information is missing or unreliable. Principal Component Analysis (PCA) is often used to describe the modes of variations among shapes to segment. PCA is reported in many studies on segmentation of bone [3789] or 
other structures [610 1112. In Bayesian approaches, the segmentation problem is formulated as a Maximum A Posteriori (MAP) problem 6 61213]. Prior knowledge is then naturally expressed as prior probabilities. Markov Random Fields (MRF) 14 express spatially varying priors and have been successfully applied to MAP-based segmentation [121315. But MAP-based methods using MRF present the inconvenient to be usually very time consuming.

Deformable models vary in type of representation (e.g., active contours [15], implicit 6 6], discrete [4101113]) and are used in segmentation techniques exploiting PCA 671011] or MRF [1315. Physically-based deformable models are particularly interesting because they can be coupled with efficient computer graphics techniques (e.g., fast physically-based simulations [1617]). The proposed approach simultaneously combines prior knowledge (PCA, MRF) and physically-based models. This novel combination confers speed and robustness to the segmentation. Unlike some MAP-PCA methods (e.g., 6]), a very fast segmentation is achieved without assuming that bone intensity is homogeneous.

In this paper, shapes are modeled as discrete deformable models by using a 2-simplex mesh representation [18]. Points of 2-simplex meshes have the nice characteristic to have the constant number of three neighbors. A shape $\boldsymbol{x}$ is represented by $M 3 \mathrm{D}$ points: $\boldsymbol{x}=\left\{x_{1}, \ldots, x_{M}\right\}$. The paper is organized as follows: shape variation modeling is first presented. Then, the segmentation method built on a multilevel forces-based implicit scheme is depicted. Finally, results of clinical MRI experiments and future work conclude this paper.

\section{Shape Variation Modeling}

\subsection{Global Shape Statistics Based on PCA}

For a given bone, $N$ training shapes with known point correspondences are aligned with respect to a common reference frame. Based on a PCA, a statistical model of the shape variations is then built [19]. In our case, shapes are the result of a supervised segmentation procedure that fits template deformable models to corresponding images. This common approach (e.g., [10]) automatically produces a direct point correspondence. Although the correspondence may not be optimal [8320], it gives satisfactory results in our experiments. An arbitrary shape $\boldsymbol{x}$ can be approximated from the computed statistics by: $\boldsymbol{x} \approx T(\overline{\boldsymbol{x}}+\Phi \cdot \boldsymbol{b})$. Vector $\overline{\boldsymbol{x}}$ is the mean shape, $\Phi$ is a matrix of $K(K<N)$ eigenvectors, $\boldsymbol{b}$ is a shape parameters vector and $T$ denotes the alignment transform. The eigenvectors (with eigenvalues $\lambda_{i}$ ) span the PCA subspace and express modes of variations. The degree of variation depends on the transform type and on the mesh resolution. Indeed, a PCA based on a rigid transform will capture global shape variations while an affine transform will lead to a more local description of variations. These two kinds of PCAs are denoted as rigid and affine PCA. In a similar way, shapes with a higher resolution are more adapted to express finer shapes differences. 


\subsection{Local Deformations Modeling Based on MRF}

Let's consider $\boldsymbol{x}=\left\{x_{1}, \ldots, x_{M}\right\}$ and $\boldsymbol{Y}=\left\{y_{1}, \ldots, y_{M}\right\}$, respectively current and true point positions of a bone shape. Positions $\boldsymbol{x}$ are commonly the result of a procedure which exploited the PCA-based modeling. The objective is now to model the last discrepancy between current and real model. We pose $\boldsymbol{Y}=$ $\boldsymbol{x}+\boldsymbol{\delta}$ where $\boldsymbol{\delta}=\left\{\delta_{1}, \ldots, \delta_{M}\right\}$ represent local deformations. By adapting the idea depicted in [12, the local deformations distribution is modeled by a first-order Gauss-Markov random process:

$$
P(\boldsymbol{\delta})=\frac{1}{Z_{m}} \exp -\frac{1}{2} \sum_{i=1}^{M}\left[\frac{1}{\eta^{2}} \sum_{j \in \mathcal{N}(i)}\left\|\delta_{i}-\delta_{j}\right\|^{2}+\frac{1}{\sigma^{2}}\left\|\delta_{i}\right\|^{2}\right]
$$

where $\mathcal{N}(i)$ denotes the indices neighborhood of point $i$ (i.e., three point indices) and $Z_{m}$ designates the partition function which is a constant. Parameters $\eta^{2}$ and $\sigma^{2}$ control the smoothness and the magnitude of the deformations respectively. The term $\boldsymbol{x}$ is considered as deterministic, $\boldsymbol{Y}$ follows hence a first-order GaussMarkov process as well. It is an unknown random process that will be inferred from observed data.

\section{Segmentation Algorithm}

\subsection{Deformable Model Evolution}

Discrete models are considered as particles with mass evolving under the Newtonian law of motion. The Newton equation relates particle position and velocity to a set of internal and external forces. The resulting time discretized differential equations system is solved by a stable implicit integration scheme 16 performing large time steps. As internal forces, we use a smoothing force 4 that penalizes strong irregularities and a PCA-based force $f^{\text {pca }}$ (Sec. 3.3) that enforces shape constraints. External forces depend on the image intensity information (Sec. 3.2) and on current mesh point positions $\boldsymbol{x}$. They are designed to move models toward anatomical boundaries. Section 3.4 explains how the MRF modeling is exploited in a scheme involving two external forces. A step of the implicit scheme consists in evaluating forces $f_{i}$ applied on each particle $i$ at position $y_{i}$, as well as derivatives with respect to position $D f_{i}=\partial f_{i} / \partial y_{i}$ and velocity $D v f_{i}=\partial f_{i} / \partial v_{i}$ (which is null for all the forces studied in this paper since they do not depend on velocity).

\subsection{Image Force}

Given the current mesh point positions $\boldsymbol{x}$, new positions $\boldsymbol{y}$ are sought in a neighborhood of $\boldsymbol{x}$ (usually along the mesh normal directions, e.g., 3[10[18]) in such a way that an energy $E_{d}(\boldsymbol{y}, \boldsymbol{d})$ is minimized. The image information $\boldsymbol{d}$ can include gradients, intensity neighborhoods, etc. A force $\boldsymbol{f}^{\mathrm{im}}$ is then derived from $\boldsymbol{x}$ and 
the computed $\boldsymbol{y}$. By assuming that forces are applied to particles $i$ independently, $\boldsymbol{f}^{\mathrm{im}}$ is modeled as a Hookean spring [18] $\boldsymbol{f}_{\boldsymbol{i}}^{\mathrm{im}}=\alpha^{\mathrm{im}}\left(y_{i}-x_{i}\right)$. The coefficient $\alpha^{\mathrm{im}}$ acts as a stiffness parameter. The derivative of the force is $D f_{i}^{\mathrm{im}}=-\alpha^{\mathrm{im}} \mathbf{I}$, where $\mathbf{I}$ denotes the identity matrix.

\subsection{PCA-Based Force}

A closest shape $\boldsymbol{y}$ is found by projecting $\boldsymbol{x}$ in the PCA space. An iterative procedure computes the adequate transformation $T$ and appropriate constrained shape parameters $\boldsymbol{b}=b_{1}, \ldots, b_{K}$. Constraints are required to discard illegal configurations: shape parameters $b_{k}$ are scaled when the Mahalanobis distance $D=\sum b_{k}^{2} / \lambda_{k}$ is strictly over a threshold $D_{\max }$. The threshold is calculated from the $\chi^{2}$ distribution with $K$ degrees of freedom [19]. The shape $\boldsymbol{y}$ is ultimately computed according to Sec. 2.1 formula. The same source-to-target approach is once again applied: $f_{i}^{\text {pca }}=\alpha^{\mathrm{pca}}\left(y_{i}-x_{i}\right)$. However, attention must be paid on the fact that the computations of $y_{i}$ and the corresponding forces are dependent on the positions of $x_{j}, j \neq i$. Forces and derivatives are in reality more complex. But neither instabilities nor odd behaviors were noticed in practice with the chosen approximations.

\subsection{MRF-Based Force}

Let's $\boldsymbol{d}=\left\{d_{t}, t \in L\right\}$ designates an observation field on a regular lattice defined by the image $I$. This field is related to the image information (Sec. 3.2). Our goal is to estimate the true (unknown) point positions $\boldsymbol{Y}^{*}$ by using a MAP formulation of the random process $\boldsymbol{Y}$ from the observation $\boldsymbol{d}[12$ :

$$
\boldsymbol{Y}^{*}=\underset{\boldsymbol{Y}}{\operatorname{argmax}} P(\boldsymbol{Y} \mid \boldsymbol{d})=\underset{\boldsymbol{Y}}{\operatorname{argmax}} P(\boldsymbol{d} \mid \boldsymbol{Y}) P(\boldsymbol{Y})=\underset{\boldsymbol{Y}}{\operatorname{argmax}} P(\boldsymbol{Y}, \boldsymbol{d})
$$

Commonly, $P(\boldsymbol{d} \mid \boldsymbol{Y})$ is assumed to follow a Gibbs distribution, i.e. $P(\boldsymbol{d} \mid \boldsymbol{Y})$ is proportional to $\exp -E_{d}(\boldsymbol{Y}, \boldsymbol{d})$. As a result, the joint distribution $P(\boldsymbol{Y}, \boldsymbol{d})$ is also a Gibbs distribution: $P(\boldsymbol{Y}, \boldsymbol{d})=\frac{1}{Z} \exp -E(\boldsymbol{Y}, \boldsymbol{d})$. Parameter $Z$ is assumed to be constant and independent of $Y[12$. The energy is expressed as:

$$
\begin{aligned}
E(\boldsymbol{Y}, \boldsymbol{d}) & =E_{d}(\boldsymbol{Y}, \boldsymbol{d})+\beta \sum_{i=1}^{M}\left(\frac{1}{\eta^{2}} \sum_{j \in \mathcal{N}(i)}\left\|\delta_{i}-\delta_{j}\right\|^{2}+\frac{1}{\sigma^{2}}\left\|\delta_{i}\right\|^{2}\right) \\
& =\sum_{i=1}^{M}\left[E_{d, i}\left(y_{i}, d_{y_{i}}\right)+\beta E_{m, i}\left(y_{i}, y_{\mathcal{N}(i)}\right)\right]=\sum_{i=1}^{M} E_{i}
\end{aligned}
$$

where $\delta_{i}=y_{i}-x_{i}$ and $d_{y_{i}}$ is the image information at $y_{i}$. The random process $Y$ will be inferred from the image information and reference positions $\boldsymbol{x}$. The parameter $\beta$ weights the regularization induced by the energy term based on spatial deformations with respect to the image-based energy term. When $\beta$ is null, the procedure can be seen as a standard Maximum-Likelihood strategy that ignores geometrical considerations. To solve this MAP problem, the energy $E$ 
should be globally minimized which is not a trivial task. Instead, we consider $E$ as a kind of potential energy (i.e., $f=-\nabla E$ ) and devise appropriate forces to be used in our framework. Each local energy $E_{i}$ is derived with respect to positions $y_{i}$ and $y_{j}(j \in \mathcal{N}(i))$, to get local contributions in forces and force derivatives. By summing up these contributions (e.g., $f_{i}=-\partial E_{i} / \partial y_{i}-\sum \partial E_{j} / \partial y_{i}$ ), forces and force derivatives can be computed on each particle in order to perform an implicit step. An analytic formulation of the energy $E_{m, i}$ derivatives can be expressed, whereas the derivative of $E_{d, i}$ cannot be explicitly formulated. Finite differences approximations may also lead to incorrect results as $E_{d}$ may not vary smoothly enough. The force expression of Sec. 3.2 is thus reused to get forces and force derivatives for $E_{d, i}\left(E_{d, i}\right.$ is not dependent on $\left.y_{j}\right)$. The MAP-MRF problem resolution yields so two types of forces: $f^{\mathrm{im}}$ and $f^{\mathrm{mrf}}$ ( $\beta$ is then similar to a weighting parameter $\left.\alpha^{\mathrm{mrf}}\right)$.

\subsection{Multilevel Segmentation Strategy}

A Thin-Plate Splines interpolation procedure initializes generic bone shapes by using landmarks placed at specific anatomical positions. During the automatic segmentation, higher mesh resolutions are successively introduced and used in combination with previous lower resolutions. This strategy uses a bottom-up forces propagation scheme [4] between the different resolutions, which linearly combines lower with higher resolution forces. This approach yields better robustness and accuracy. At each new higher resolution introduction, three successive steps take place: (i) exploit PCA, image and smoothing forces; (ii) reduce PCA influence and keep other forces; (iii) set the current position as reference, enable MRF-based force, disable PCA and smoothing. The weight of each force is controlled by the weight factors $\alpha$. Low resolution models are coupled with rigid PCA, whereas affine PCA is reserved for higher resolutions. MRF-based force is not used at the first resolution as the final result may be too far. In fact, only convergence toward a local minimum is ensured, so models must be close enough to the final solution.

\section{Experiments and Results}

An axial 3D T1 protocol $(\mathrm{TR} / \mathrm{TE}=4.15 / 1.69 \mathrm{~ms}, \mathrm{FOV} /$ Matrix $=35 \mathrm{~cm}, 256 \times 256$, resolution $=1.367 \times 1.367 \times 5 \mathrm{~mm})$ is used to acquire a large volume covering hips and thighs from a 1.5T MRI device (Philips Medical Systems). The protocol is fast (acquisition time: $3 \mathrm{mn}$ ) but low resolution images are produced (Fig. 11). An experiment consists in segmenting hip and femur bones of both sides (Fig. 1(d)) from a volume dataset. Test datasets are not used in the training shapes construction. A set of 29 right and left female training bones is used, each training shape is available in 4 different resolutions ( femur (hip) bone number of vertices: 514 (814) to $32 \mathrm{~K}(52 \mathrm{~K})$ ). A rigid PCA is performed for the lowest resolution, and affine PCAs for the remaining resolutions. $99 \%$ of the total variance is kept for each PCA. In all the experiments, we set $1 / \eta^{2}=0.15$ and $1 / \sigma^{2}=0.25$. The manual initialization requires the placement of 6 markers 
Table 1. Mean and standard deviation of error distance with computation time

\begin{tabular}{|l||c|c|c|c|c|c|}
\hline measures / dataset \# & 1 & 2 & 3 & 4 & 5 & 6 \\
\hline mean $(\mathrm{mm})$ & 1.66 & 1.34 & 1.30 & 1.50 & 1.27 & 1.54 \\
\hline std $(\mathrm{mm})$ & 1.25 & 0.82 & 1.15 & 1.20 & 0.95 & 1.22 \\
\hline time $(\mathrm{s})$ & 162 & 165 & 162 & 163 & 161 & 166 \\
\hline
\end{tabular}
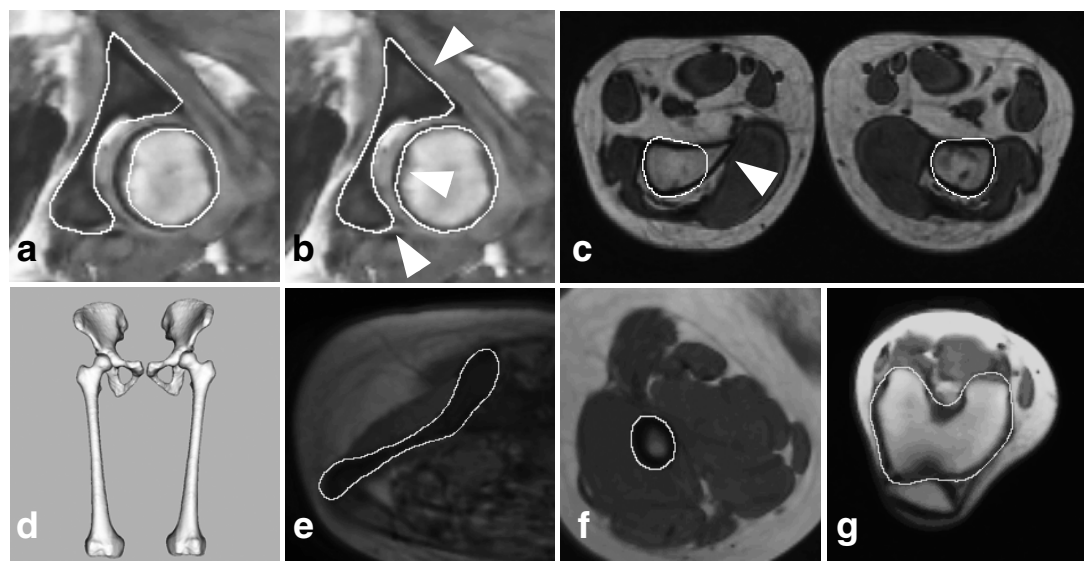

Fig. 1. Segmentation results: before (a) and after (b) usage of $f^{\text {mrf }}$, white arrows indicate significant improvements $\left(\alpha^{\mathrm{im}}=\alpha^{\mathrm{mrf}}=0.5\right)$. (c) Error in segmentation in case of abnormal deformation. Patient \#1 has a benign osteochondrome (cartilage tumor) at the right distal femoral metaphysis (white arrow). (d) 3d reconstructed models example. (e)-(g) axial slice examples with segmentation contours.

per bone. This requires about 4-5mn in total. Segmentation error is calculated from experts contours (sometimes reduced to a series of points if contours cannot be drawn with a good confidence). Each contour point is projected on the corresponding segmented shapes and Euclidean distances are computed. Execution time of a segmentation (initialization is not counted) is monitored (used equipment: $3.40 \mathrm{Ghz}$ computer with $2 \mathrm{~Gb}$ of RAM). Table 1 reports accuracy and computation time for 6 datasets.

The average error is $1.44 \pm 1.1 \mathrm{~mm}$, which is close to the axial resolution $(1.37 \mathrm{~mm})$, and visually the segmentation looks accurate (Fig. 1). Nevertheless, a little too high standard deviation reveals some noticeable errors, essentially due to two major factors. Firstly, the poor image quality may also corrupt the gold standard manual editing, experts segmentation remains error prone. Secondly, the segmentation may fail in very noisy areas or when a bone presents a strong deviation from the training shapes. In that case, the segmentation is "over-constrained" by the priors-based forces (Fig. 1(c)). But, PCA-based forces make the process less sensitive to noise and surroundings structures (Fig. 2), the absence of PCA "regularization" creates in fact an unstable model (Fig. 2(b)). The MRF-based force efficiency is illustrated in Fig. 1(a) and 1(b), where at 

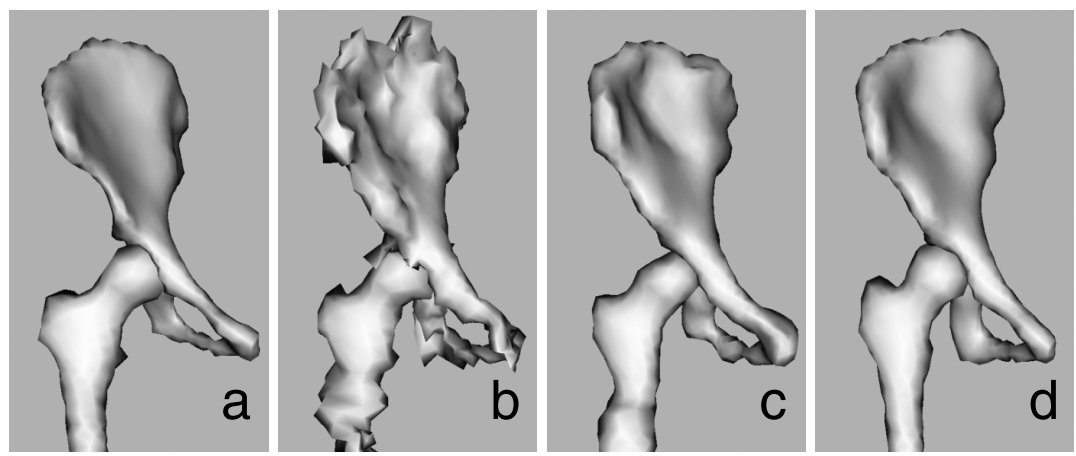

Fig. 2. PCA-based force utility: Starting from an initial model at low resolution (a) in which bones are deformed, 80 iterations are performed with different kind of forces. (b) $\alpha^{\text {pca }}=0, \alpha^{\text {smo }}=0, \alpha^{\text {im }}=1(\mathrm{c}) \alpha^{\text {pca }}=0, \alpha^{\text {smo }}=0.5, \alpha^{\text {im }}=0.5(\mathrm{~d}) \alpha^{\text {pca }}=0.4, \alpha^{\text {smo }}=$ $0.2, \alpha^{\mathrm{im}}=0.4$. The usage of PCA-based force clearly gives the best result.

the second mesh resolution level, the force can already capture fine details (in this ex., the error decreases from $1.94 \pm 1.7$ to $1.62 \pm 1.5)$. Last but not least, time is also a strength of the method: overall time (acquisition, initialization and segmentation) takes less than 10mn. This encourages clinical use as image resolution and time constraints are not too demanding.

\section{Conclusion and Future Work}

The proposed method showed how prior knowledge supported by PCA and MRF brings robustness and accuracy, when they are combined with fast physicallybased deformable models. Still, work remains in considering pathologies or large patient anatomical differences that are discarded by the priors-based regularization. One direction would be to add in the training shapes these differences or to balance correctly image forces with respect to other forces. Finally, the method should be adapted to higher resolution clinical datasets that cover a smaller field (i.e., focused on a joint), by tackling the issue of a more automatic initialization.

Acknowledgments. This work is supported by the 3D Anatomical Human project funded by the European Union. We would like to thank Dr. KoloChristophe from the Geneva University Hospital and Pascal Volino for their collaboration.

\section{References}

1. Felson, D.: Clinical Practice. Osteoarthritis of the Knee. N. Engl. J. Med. 354, 841-848 (2006)

2. Pfirrmann, C.W.A., Mengiardi, B., Dora, C., Kalberer, F., Zanetti, M., Hodler, J.: Cam and Pincer Femoroacetabular Impingement: Characteristic MR Arthrographic Findings in 50 Patients. Radiology 240(3), 778-784 (2006) 
3. Fripp, J., Crozier, S., Warfield, S., Ourselin, S.: Automatic Segmentation of the Bone and Extraction of the Bone-cartilage Interface form Magnetic Resonance Images of the Knee. Phys. Med. Biol. 52, 1617-1631 (2007)

4. Gilles, B., Moccozet, L., Magnenat-Thalmann, N.: Anatomical modelling of the musculoskeletal system from MRI. In: Larsen, R., Nielsen, M., Sporring, J. (eds.) MICCAI 2006. LNCS, vol. 4190, pp. 289-296. Springer, Heidelberg (2006)

5. Lorigo, L.M., Faugeras, O.D., Grimson, W.E.L., Keriven, R., Kikinis, R.: Segmentation of Bone in Clinical Knee MRI using Texture-based Geodesic Active Contours. In: Wells, W.M., Colchester, A.C.F., Delp, S.L. (eds.) MICCAI 1998. LNCS, vol. 1496, pp. 1195-1204. Springer, Heidelberg (1998)

6. Yang, J., Duncan, J.S.: 3d image segmentation of deformable objects with joint shape-intensity prior models using level sets. Med. Image Anal. 8, 285-294 (2004)

7. Leventon, M.E., Grimson, W.E.L., Faugeras, O.: Statistical shape influence in geodesic active contours. In: Proc. IEEE Conf. Comput. Vis. Pattern Recogn., vol. 1, pp. 316-323 (2000)

8. Lamecker, H., Seebaß, M., Hege, H.C., Deuflhard, P.: A 3d statistical shape model of the pelvic bone for segmentation. In: Proc. of the SPIE, vol. 5370, pp. 1341-1351 (2004)

9. Dong, X., Gonzalez Ballester, M.A., Zheng, G.: Automatic extraction of femur contours from calibrated x-ray images using statistical information. J. Multimed. 2(5), 46-54 (2007)

10. Costa, M., Delingette, H., Novellas, S., Ayache, N.: Automatic segmentation of bladder and prostate using coupled 3d deformable models. In: Ayache, N., Ourselin, S., Maeder, A. (eds.) MICCAI 2007, Part I. LNCS, vol. 4791, pp. 252-260. Springer, Heidelberg (2007)

11. Wang, Y., Staib, L.: Physical model-based non-rigid registration incorporating statistical shape information. Med. Image Anal. 4(1), 7-20 (2000)

12. Kervrann, C., Heitz, F.: A hierarchical markov modeling approach for the segmentation and tracking of deformable shapes. Graph. Model. Image Process 60(3), 173-195 (1998)

13. Huang, R., Pavlovic, V., Metaxas, D.N.: A graphical model framework for coupling mrfs and deformable models. In: Proc. Conf. Comput. Vis. Pattern Recogn (CVPR 2004), vol. 02, pp. 739-746 (2004)

14. Geman, S., Geman, D.: Stochastic relaxation, gibbs distributions, and the bayesian restoration of images. IEEE Trans. Pattern Anal. Mach. Intell. 6, 721-741 (1984)

15. Martín-Fernández, M., Alberola-López, C.: An approach for contour detection of human kidneys from ultrasound images using markov random fields and active contours. Med. Image Anal. 9(1), 1-23 (2005)

16. Volino, P., Magnenat-Thalmann, N.: Implementing fast cloth simulation with collision response. In: Proc. Int. Conf. on Computer Graphics (CGI 2000), pp. 257-266. IEEE Computer Society, Los Alamitos (2000)

17. Nealen, A., Müller, M., Keiser, R., Boxerman, E., Carlson, M.: Physically based deformable models in computer graphics. Computer Graphics Forum 25(4), 809 836 (2006)

18. Delingette, H.: General object reconstruction based on simplex meshes. Int. J. Comput. Vis. 32(2), 111-146 (1999)

19. Cootes, T.F., Hill, A., Taylor, C.J., Haslam, J.: The use of active shape models for locating structures in medical images. In: Barrett, H.H., Gmitro, A.F. (eds.) IPMI 1993. LNCS, vol. 687, pp. 33-47. Springer, Heidelberg (1993)

20. Davies, R.H., Twining, C.J., Cootes, T.F., Waterton, J.C., Taylor, C.J.: 3d statistical shape models using direct optimisation of description length. In: Heyden, A., Sparr, G., Nielsen, M., Johansen, P. (eds.) ECCV 2002. LNCS, vol. 2352, pp. 3-20. Springer, Heidelberg (2002) 